Revue

Revue de l'histoire des religions

de Ihistoire des religions

3 | 2008

Varia

\title{
Andreas Merkt, Das Fegefeuer. Enstehung und Funktion einer Idee
}

Charlotte Touati

(2) OpenEdition

Journals

Édition électronique

URL : http://journals.openedition.org/rhr/6703

DOI : $10.4000 /$ rhr.6703

ISSN : 2105-2573

Éditeur

Armand Colin

Édition imprimée

Date de publication : 1 septembre 2008

Pagination : 419-423

ISBN : 978-2200-92445-4

ISSN : 0035-1423

Référence électronique

Charlotte Touati, «Andreas Merkt, Das Fegefeuer. Enstehung und Funktion einer Idee », Revue de l'histoire des religions [En ligne], 3 | 2008, mis en ligne le 14 janvier 2010, consulté le 22 septembre 2020. URL : http://journals.openedition.org/rhr/6703 ; DOI : https://doi.org/10.4000/rhr.6703 
pour l'étude de l'Antiquité. On a déjà mentionné la peinture pompéienne et le décor de la Domus aurea, mais l'archéologie et la sculpture ne sont pas non plus négligées : les nouvelles découvertes sur le chef-lieu de la cité des Leuques viennent enrichir un dossier utilement présenté, une statue de Vaison-la-Romaine fait l'objet d'une nouvelle description et un bloc de calcaire retrouvé à Reims et représentant une femme donnant le sein complète la documentation iconographique de la Reims antique.

L'épigraphie est abondamment représentée et parfois de manière spectaculaire : un nouveau poids de plomb jette un nouvel éclairage sur le dossier de L. Egnatius Victor Lollianus. Alliée aux sources littéraires, l'épigraphie permet aussi de connaître les dévotions privées des gouverneurs de province et de distinguer la nature d'Aiôn dans deux documents pourtant contemporains. L'intérêt de comparaisons entre les diverses sources ressort clairement de l'étude minutieuse des représentations antiques du lièvre ; complétée d'une analyse anthropologique des sacrifices sanglants dans le monde celte, cette étude conduit à minimiser l'importance de la rhétorique dans la description des massacres perpétrés par Boudicca.

Les familiers de l'œuvre de Jean-Pierre Martin ne seront pas surpris de trouver aussi dans ce volume deux communications consacrées à la numismatique : un brillant article de synthèse et de méthode consacré aux rapports de la politique et de la religion dans le monnayage de la fin de la République (qu'on pourra enrichir des réflexions sur la libération typologique du denier présentées par Jonathan Williams et Andrew Meadows in Journal of Roman Studies 91, 2001, 27-49) et une analyse du type du laboureur (qui aurait sans doute gagné à s'appuyer sur les documents de travail les plus récents et non sur le vieux catalogue de Grueber). Ce volume correspond donc admirablement à son dédicataire : on y traite avec pénétration de la religion et du pouvoir, en exploitant avec bonheur toutes les branches de l'histoire ancienne.

Arnaud SUSPENE, Université d'Orléans/IRAMAT-Centre Ernest-Babelon.

Andreas MERKT, Das Fegefeuer. Enstehung und Funktion einer Idee, Darmstadt, Wissenschaftliche Buchgesellschaft, 2005, 21,5 cm, 131 p., $29,90 €$ €.

A. Merkt donne le ton dès l'introduction et expose buts et motivations avec une clarté réjouissante. Ainsi, le nom de Jacques Le Goff apparaît à la première ligne, en-tête programmatique. Un historien ne peut, même un quart de siècle après sa parution, aborder la question du purgatoire sans se prononcer sur La Naissance du Purgatoire (Paris, 1981). Il peut en revanche s'appuyer sur l'autorité de J. Le Goff pour ensuite se positionner et organiser sa matière. Selon A. Merkt, J. Le Goff raisonne en médiéviste. 
Un purgatoire localisé, le troisième espace de l'au-delà, est une pure réalité médiévale. Ce purgatoire-là n'émergeant qu'au douzième siècle, toutes les manifestations antérieures n'appartiendraient qu'à sa préhistoire.

Le dossier est alors rouvert avec un regard d'antiquisant porté sur l'Afrique du Nord entre 180 et 250 après J.-C. De surcroît, l'objet demande à être redéfini, sa localisation n'étant plus un élément constitutif. L'historien de l'Antiquité est également en droit de soulever des questions subsidiaires. Les liens unissant purgatoire et suffrages des vivants sont-ils indissolubles et fondamentaux ? Le purgatoire est-il si intimement chrétien, occidental et latin qu'on ne puisse documenter des parallèles et même des emprunts aux religions non-chrétiennes, ou simplement non-catholiques ? Si la naissance du purgatoire s'accompagne de mutations dans les champs du savoir et de l'histoire, les transformations de l'Empire autour des règnes sévériens n'ont-elles pas vu également leur lot d'innovations ? Autant de questions stimulantes qui charpentent l'étude d'A. Merkt.

L'auteur explique le choix de son corpus en constatant que depuis le Moyen Âge, l'existence du purgatoire fait débat et oppose les tenants de la foi catholique latine aux chrétiens orientaux, puis aux protestants. Ce contexte polémique a largement modelé l'approche des textes patristiques et ce, jusqu'à nos jours. Sur cette base méthodologique, l'auteur entame une série d'études textuelles. Après une brève mise en contexte de la Passion de Perpétue et de Félicité, le champ est restreint à deux visions où la future martyre voit son défunt frère, souffrant de sa situation au-delà. Perpétue s'abîme alors dans la prière et parvient à le tirer de ses tourments. En détaillant les nombreuses interprétations suscitées par les visions de Perpétue, A. Merkt signale à nouveau son souci de s'inscrire dans la recherche, de dialoguer avec elle et de compléter ce qui fut négligé jusqu'alors. Les différentes analyses sont classées selon le point de vue adopté et chaque groupe donne l'occasion d'un résumé discursif, clair et essentiel des problématiques. La chronologie des contributions (toutes les références sont bien entendu reportées en notes) est gommée, évitant ainsi la sécheresse d'un catalogue. Chaque perspective connaît ses limites qu'A. Merkt pointe pour mieux relativiser la portée d'une explication unique du texte. Sa position personnelle est énoncée dans un second temps, pas de la manière attendue, mais selon une démonstration par l'absurde : si aucune hypothèse n'a donné le fin mot des visions de Perpétue, c'est qu'on a voulu leur faire dire davantage qu'elles ne peuvent signifier.

Avec Tertullien, le sort des défunts est abordé dans et par un esprit différent de celui de Perpétue. Ses textes sont beaucoup plus explicites. A. Merkt expose alors de manière synthétique le traitement de trois problèmes par Tertullien : la situation des âmes entre mort et résurrection, le délai de la résurrection et l'exégèse de Mt 5,25. Les conceptions éthiques et eschatologiques du maître ont évolué au cours de sa vie et de son œuvre, mais l'Adversus Marcionem, le De anima et le De resurrectione permettent d'apprécier l'essentiel de ses représentations de l'après-vie, marquées par 
un souci pénitentiel qui travaillera également son compatriote Cyprien. Ce dernier n'a certainement pas lu les ouvrages de son prédécesseur frappé d'une véritable damnatio memoriae pour cause de montanisme. Le traitement de problèmes similaires par l'évêque et par le quasi hérétique mérite que l'on s'y attarde. Concernant la situation de l'âme entre mort et résurrection, Cyprien ne professe pas l'extrême rigorisme de Tertullien et semble ouvrir le repos éternel à d'autres qu'aux martyrs. Un jugement personnalisé à la fin des temps est exclu, ce qui laisse supposer l'existence d'une période perfective entre la mort individuelle et le jugement collectif. Mais le propos de Cyprien est beaucoup moins explicite que ne le fut celui de Tertullien. Peut-être est-ce la prolixité de ce dernier et l'association de son nom à l'interim qui incitent Cyprien à une certaine discrétion. On pourrait dès lors s'interroger sur l'existence même de cet interim dans son système.

Une purification post-mortem est attestée dans une lettre à Antoninianus, fruit de la querelle contre Novatien, « anti-pape » refusant de réintégrer les lapsi au sein de l'Église. Interrogé sur la question par son confrère Antoninianus, Cyprien démontre qu'il n'y a pas danger pour la communauté à accueillir ceux qui ont chuté, moyennant pénitence. C'est alors qu'il oppose le sort de ces pénitents, qui paieront encore dans l'au-delà pour s'être dérobés à la prison et à la mort, à celui des martyrs qui ont affronté la violence terrestre, mais sont ensuite libérés des tourments dans la mort. L'affirmation d'une pénitence post-mortem a parfois concouru à ériger Cyprien en père du purgatoire. À partir d'une étude lexicale, A. Merkt démontre la portée ecclésiologique de cette missive, souvent occultée au profit d'une lecture eschatologique. En effet, l'ancrage historique du débat implique de s'interroger sur l'Église, les fins dernières, le pardon humain et divin, autant de questions vitales situées à des registres différents, mais fortement interdépendantes. Une synthèse intermédiaire reprend les caractéristiques essentielles d'un purgatoire qui semble poindre à Carthage au début du troisième siècle. A. Merkt relève que Cyprien double la métaphore de la prison par celle du feu et qu'il est le premier auteur à user du verbe purgare.

Dans la première partie de l'étude, le corpus était présenté de manière chronologique, en soulignant les points communs ou les spécificités des textes, ainsi que le contexte historique qui les a vu naître. Dans la seconde partie, les mêmes œuvres sont traitées de manière transversale, par une série d'études thématiques. Les prières ou offrandes des vivants en faveur des défunts ouvrent la danse. Rituel d'apaisement ou rite propitiatoire, la prière païenne aux morts a pour bénéficiaire celui qui la prononce et non l'âme invoquée. La prière d'intercession destinée au défunt lui-même serait donc caractéristique du christianisme. Autre conclusion majeure de la seconde partie : intervenir dans le sort des défunts implique un stade intermédiaire entre enfer et paradis, un purgatoire qui brise l'image d'un au-delà bipartite et inamovible.

Après avoir examiné la relation des vivants aux morts dans sa dimension individuelle (l'épouse prie pour l'époux, la sœur, pour le frère), A. Merkt 
s'intéresse à la partie collective ou ecclésiale du dossier. Au troisième siècle, le christianisme est en passe de devenir une religion populaire, mais la composition des communautés laisse parfois à désirer. Lucide, Cyprien admet que nul ne peut se reconnaître la capacité de séparer le bon grain de l'ivraie sans une exorbitante présomption. Seul dieu sonde les âmes ; il lui revient donc de passer l'ecclesia au crible, ou, selon les dires de l'évêque, de la passer au feu. Aussi importante que la mention du purgatoire comme lieu, la prise de conscience que tous les baptisés ne sont pas du métal le plus pur et que l'Église terrestre n'est pas la Jérusalem céleste pourrait marquer l'acte de naissance du purgatoire.

Carthage voit l'affirmation d'un autre phénomène d'importance : un légalisme de tendance montaniste, dont Tertullien est le plus fameux porte-parole. L'âme doit s'acquitter de toute dette dans l'au-delà, jusqu'au dernier denier ; jusqu'au dernier péché qu'elle soit purgée par le feu. À ce légalisme, A. Merkt oppose les conceptions intériorisées des deux Alexandrins Clément et Origène. Marqués par le platonisme, ils décrivent un feu plus purificateur que punitif, au cœur d'une dynamique positive d'éducation de l'âme.

Dernier volet, A. Merkt met en équation purgatoire et histoire, selon deux conceptions : apocalyptique et gnostique. La doctrine du purgatoire semble incompatible avec la croyance en une fin des temps imminente qui aurait le feu du jugement en ligne de mire. Mais au troisième siècle l'urgence apocalyptique s'est refroidie. La fin du monde n'est plus pour demain, ce qui laisse quelque délai pour développer une eschatologie individuelle. En envisageant le temps des tribulations (celui du feu purgatoire) comme un emprisonnement, Tertullien se distingue de la conception gnostique de l'histoire, qui considère que la prison n'est pas l'Hadès, mais le monde lui-même dans toutes les dimensions qui le régissent, temps et espace.

A. Merkt considère ensuite la piste du judaïsme rabbinique. À plus d'un titre intéressante, elle présente l'avantage de croiser l'itinéraire africain tracé auparavant. Perpétue, Tertullien ou Cyprien ont pu entrer en contact avec le judaïsme, attesté dans la province d'Afrique. Au nombre des représentations communes avec le christianisme, on pourra citer l'existence d'un au-delà temporaire, la division du séjour souterrain en deux étages, le plan supérieur accueillant les défunts en attente de la miséricorde divine. L'interconnexion des cultures et des religions dans la koiné est une des clés du problème, réservant quelques surprises : même le néo-platonisme présente des traits que l'on croirait propres à la tradition judéo-chrétienne.

La discrétion des prises de position personnelles d'A. Merkt pourrait surprendre, mais c'est oublier sa méthode. En retraçant l'histoire de la recherche, il fait coup double. Une hypothèse n'est jamais discutée pour elle-même, mais seulement dans les aspects utiles au plan d'ensemble. En d'autres termes, il annonce les problématiques en citant des travaux antérieurs, dont il valide ou non les conclusions, jusqu'à ce que le champ soit balisé. C'est l'intelligence des hypothèses retenues, de leur nombre, de 
leur extension et des interactions possibles qui laisse affleurer l'originalité de l'étude d'A. Merkt.

Charlotte TOUATI, Université de Lausanne.

Lenka JiroušKovÁ, Die Visio Pauli. Wege und Wandlungen einer orientalischen Apokryphe im Lateinischen Mittelalter, unter Einschlu $\beta$ der alttschechischen und deutschsprachigen Textzeugen, Leiden, 2006, Brill, 24 cm, XVI + 1033 p., $206 €$ (Mittellateinische Studien und Texte, 34).

Ce volume de Lenka Jiroušková apporte une contribution nouvelle et originale à l'étude du texte de la Visio Pauli. Il contient une recension de toute la tradition manuscrite latine et la première édition complète des différentes versions latines de ce texte. La Visio Pauli est une version abrégée de l'Apocalypse de Paul, un texte apocryphe écrit en grec vraisemblablement vers la fin du quatrième siècle, à ne pas confondre avec le livre homonyme retrouvé dans la bibliothèque gnostique de Nag Hammadi. Le texte dont il est question ici relate le voyage que l'apôtre Paul aurait fait dans l'au-delà, selon un passage de la deuxième épître aux Corinthiens dans lequel l'auteur raconte son ravissement jusqu' au troisième ciel (2 Cor. 12, 2-5). Dans l'original grec de l'Apocalypse de Paul, ce voyage comporte tant une vision de l'enfer que du paradis, mais cette version du texte fut bientôt supplantée en milieu latin par notre rédaction courte, qui rapporte seulement le voyage dans l'au-delà infernal. Il s'agit en effet de plusieurs rédactions abrégées, qui ont largement circulé dans l'occident latin pendant tout le Moyen Âge. La diffusion de la Visio Pauli a été très large, car il s'agissait sans doute du texte le plus connu et le plus ancien de l'ensemble de la littérature latine des « visions » de l'au-delà, qui trouve dans la Comédie de Dante Alighieri son couronnement littéraire. Ce n'est donc pas un hasard si Francesco Buti, vers la deuxième moitié du XIV ${ }^{\mathrm{e}}$ siècle, au moment de commenter le passage de Enfer II, 13-33, soutenait déjà que Dante était informé du récit apocryphe qui racontait la visite de Paul dans le monde infernal.

Ce volumineux travail, fruit d'une thèse de doctorat, est divisé en deux parties, une première monographique et une seconde comportant l'édition du texte. Étant donnée la richesse de ce volume, je me contenterai de donner ici une présentation systématique de son contenu, en renvoyant toute remarque technique à une future note à paraître dans la revue Adamantius. La première section du livre contient une étude de toute la tradition textuelle latine, qui prend en compte pour la première fois l'ensemble des 113 manuscrits, dont les 7 comportant un texte long (avec la vision de l'enfer et du paradis). Au catalogue de la tradition latine ainsi dressé 